\title{
The Lathe with Hinge Thread Taper Shank of Research
}

\author{
Tinghui Ma \\ Dalian Vocational \&Technical College, Dalian, China \\ mth5166@126.com
}

\begin{abstract}
The rapid development of the manufacturing industry today, for the thread machining production increasingly requires high precision, and the accuracy of thread processing is influenced by many factors. For external thread processing in large quantities, in order to improve the efficiency and accuracy of thread, research and design a kind of hinge thread tools can be installed on the tailstock of a lathe, methods to replace the existing traditional hand operation hinge thread. It is economical and practical as one of the hinge thread tool.
\end{abstract}

Keywords-Hand Operation; Lathe; Hinge Thread; Taper Shank; Screw Die; Precision; Research.

\section{INTRODUCTION}

At present, in the machining process, the actual production process, Thread cutting is the best processing method of thread processing a maximum efficiency, economy, generally refers to the molding tool or tool on the workpiece processing thread, mainly turning, milling, tapping, silk. Turning, milling and grinding thread per revolution of the workpiece turns, the machine drive chain assurance tools, milling or grinding wheel along the axial direction of the workpiece accurately and uniformly mobile a guide process. In the tapping and threading. (tap or die) tool and the workpiece for relative rotational movement, and by the formation of the first thread groove guide tool (or workpiece) moved in the axial direction. when the use of lathe turning thread parts, generally the large diameter of external threads can be turned with external thread lathe tool, and small diameter of external threads is very difficult with the external thread lathe tool to complete, while the use of the hinge bar process the thread is almost the only processing method. the hinge thread is called for machining internal thread with screw die, this is most a widely applied external thread of processing method. But with hand operation hinge thread does exist a lot of problems. So we only adopt the hinge thread of correctly method, in order to guarantee hand operation with hinge thread machining quality. This traditional method of hand operation with hinge thread is low work efficiency, hinge the thread with the workpiece perpendicularity errors is very large, also with the hand operation the stress vary in size, often appear the screw die chipping, thread accuracy low, length of thread processing is also very difficult to control. And it is not suitable for mass production, so in the actual mass production, it's mainly adopts the hinge thread with the machine. To ensure that the workpiece hinge thread quality and improve production efficiency, reduce production cost. But in the use of machine process hinge thread, it must also be the proper use of equipment and auxiliary tools, if not properly use equipment and auxiliary tools, it will also affect the processing precision of the workpiece on the thread.

The existing problems in between the above technology, research and design a new hinge thread taper shank at the lathe, can be the design of taper shank portion inserted into the taper hole of lathe tailstock inner, using ordinary lathe machine small diameter of external thread, so as to overcome the problems existing in the prior art.

\section{Analysis Of The Problems In The Process OF HAND OPERATION HINGE THREAD}

Manual threading is used to die in the bar (or pipe material workpiece with outer screw threads, usually only the small outside diameter of thread that circular screwing die processing. Precision machining accuracy depends on the set of wire to die,the hand operation hinge thread will appear in many practical problems in the production process:

1) The hand operation hinge thread often screw die face and being round rod hinge thread axis is very difficult to guarantee the full vertical. This process out of the thread have be depth on the two sides, and some even appear rotten teeth situation;

2) The hand operation hinge thread before because of screw die end face and round rod hinge screw axis is not perpendicular and repeatedly corrected, so that the partial thread cut off too much, resulting in smaller middle diameter of the thread (tooth shaped thin);

3) The hand operation hinge thread commonly put the workpiece is clamped in a parallel jaw, so it is easy to appear to clamp deflection and clamp mark;

4) The hand operation hinge thread processing, the operation when the hard uneven, the hinge bar easily skew, thread processing after the deflection;

5) The hand operation hinge thread processing, overexert, hinge bar master instability, easy to damage the screw die.

\section{DESIGN OVERVIEW}

The design scheme of the lathe with hinge thread taper shank, the characterized with the lathe with hinge thread taper shank is composed of taper shank 1、 guide bar 2 、 screw die case 3、 guide block8 and other components; the taper hole of lathe tailstock for Morse No. 5 cone, the design scheme of the taper shank 1 accurately inserted into the taper hole lathe tailstock inner; taper shank 1 is processed with a threaded hole, through a fixed bolt 5 the taper shank1 and guide bar 2 connected together, a guide bar 2 inner hole and the other end of the taper shank 1 is in clearance fit with the gap to ensure, in $0.10 \mathrm{~mm} \sim 0.20 \mathrm{~mm}$ between, in order to 
guarantee the processing thread form and position tolerance requirements; the guide bar 2 is processed with a guide groove 7 , with the fastening bolt 9 will guide block 8 fixed guiding groove 7 and taper shank 1 connected together, a guide block 8 in the guiding groove 7 along the axis direction of the sliding; the guide block 8 with scale indication arrows; on the guide bar 2 above guide groove 7 scale graduation line 11 , it can be observed at any time the depth of processing screw thread; a screw die case which is processed with a threaded hole 6 for screw die 4; the screw die 4 of diameter size is different so cannot be assembled with the guide bar 2, through the screw die case 3 will be two with fixed bolts 5 are connected together, and a fixing bolt 5 spin to screw die positioning groove 10 .

The design scheme of a total of 3 drawings, including:

Figure 1 is the main design plan of optic local profile structure schematic diagram;

Figure 2 to figure 1 the left optic structure schematic diagram;

Figure 3 is design the schematic diagram of the main structure;

In the picture: 1、 taper shank 2、 guide bar 3、screw die case 4、screw die 5、fixed bolt 6、threaded hole 7 、 guide groove 8 、 guide block 9、fastening bolt 10、 screw die positioning groove 11、 graduation line

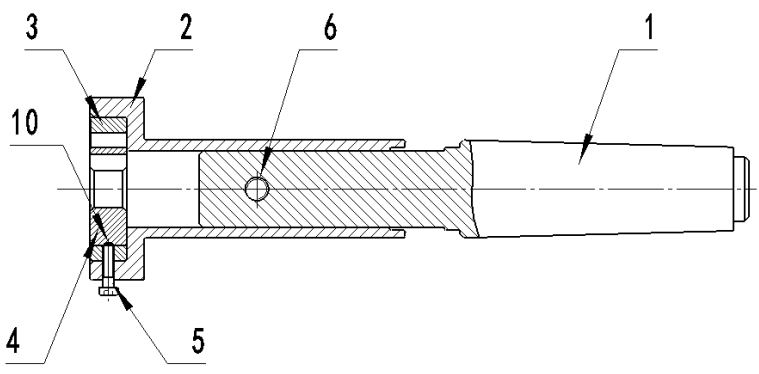

Figure 1

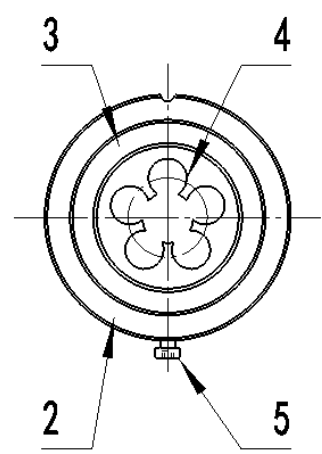

Figure 2

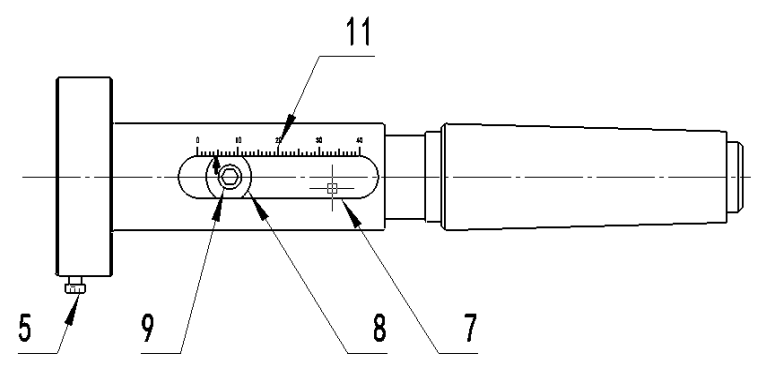

Figure 3

\section{THE SPECIFIC IMPLEMENTATION WAY}

The design scheme of the specific implementation way is first hinge thread taper shank inserted into the taper hole of lathe tailstock, the lathe tail block pull to processing thread to the appropriate location, rotating tailstock handle, screw die pressure to be processed on site, in order to facilitate the screw die cut into the workpiece, at this time it should be observed screw die end face and the round rod axis is vertical, hinge wire before the end of round bar should process chamfer, and chamfer diameter should be less than the thread. Clean the die chip groove and the thread scraps. Hinge screw cutting torque is large, circular rod workpiece should use hardwood V type clamping block or thick copper liner is fixedly clamped, can firmly clamp. In the process, join some cutting fluid to improve the accuracy of thread. Then you can start the lathe after the upward lift lever, the lathe spindle low forward, this guide block in the guide groove axial movement, can be observed at any time scale change value, To know that at the processing thread length, a breakthrough after 4 laps, no longer applied axial force, let die natural introduction, so as not to damage the screw die and have good processing thread, and it will often should reverse the chip breaking. The thread length when machined to the required when pressing down the joystick, the lathe spindle reversal screw die from workpiece, thread when the processing of different specifications, we use different screw die, at the same time it should match different of screw die case.

In addition, the tools work when it adopts machine hinge thread, so the use of cutting fluids in machining processes is very important, first is to ensure adequate cutting fluid, and the cutting fluid selection is also very important, on the plastic material need enough cutting fluid, the general use of emulsified oil or sulfide cutting oil, if be processing thread on the workpiece surface roughness value demand is not very high, but with rapeseed oil and molybdenum disulfide, soybean oil the effect is relatively good.

\section{The Advantages Of This Design Scheme}

Advantages of this design Scheme is obviously, mainly in the:

1) Due to the use of the design scheme, only need a fixed position, you can put out the thread processing, greatly improving the production efficiency; 
2) Due to the use of the design scheme, after the thread processing improve the tolerances of form and position accuracy;

3) Due to the use of the design scheme, it is processing thread uniform stress, the tooth type is high quality;

4) Due to the use of the design scheme, can effectively control the thread length;

This design scheme has the advantages of novel structure, simple processing, convenient use, improve work efficiency, improve product precision, the mass marketing will have a positive social benefits and remarkable economic benefit.

\section{SUMMARY}

In the end, using this die fixture, won't produce plate braces cone and tailstock hole slip phenomenon, causing the tailstock sleeve wear, also has the advantages of simple manufacture, less material consumption, production cost saving die fixture, thread more convenient, fast and efficient, set cut the thread of effective length also longer than the actual set the thread, tailstock sleeve inner hole is provided with a deep, you can processing a long thread, in practical work, also made good processing effect. The design scheme of lathe hinge thread taper shank function is obvious, mainly in the increase in processing speed, reduce the auxiliary time, especially in mass production when the more obvious; after processing the verticality error of thread and the workpiece can be assured, to avoid deviation phenomenon in hand operation hinge thread, in processing in the process of uniform stress, avoid the die edge collapse, the thread tooth phenomenon can be observed at any time to be processed; the length of the thread, easy to guarantee the processing length of thread.

In the path of thread processing, in order to overcome the drawbacks of the current manually hinge wire operation method, research and design of the hinge wire matching tool model. Through the foundation in machining tool on the existing structure, reasonable configuration, so as to improve the machining precision, the external thread to high-quality, high efficiency, low energy consumption structure design to finish the assembly process and product parts, this also acts as technical staff continue to explore, continue to study the direction.

\section{REFERENCES}

[1] Yao Di. Manual tapping practice problem analysis. [J]. Value Engineering.2012(21):34-36

[2] Ja Qiang. Analysis of fitter thread processing technology. [J]. science and technology innovation and Application. 2013(29):135

[3] Zhao Yongchun. The thread processing method to explore. [J]. science technology and Industry.2014, 13(9):87-88 